ARTICLE OPEN

Check for updates

\title{
Oiling-out effect improves the efficiency of extracting aroma compounds from edible oil
}

\author{
Daisuke Suzuki $\mathbb{D}^{1,2 凶}$, Yuko Sato ${ }^{1}$, Hiroshi Kamasaka ${ }^{1}$, Takashi Kuriki ${ }^{1}$ and Hirotoshi Tamura ${ }^{2,3}$
}

Volatile compounds in foods are a significant factor that affects food intake and preference. However, volatile components in edible oils are poorly understood due to a strong matrix effect. In this study, we developed a method of extracting volatile compounds from extra virgin coconut oil (EVCO) by means of oiling-out assisted liquid-liquid extraction (OA-LLE). Consequently, 44 aroma compounds were isolated and identified from only $5 \mathrm{~g}$ of EVCO. Various aroma compounds were detected in addition to $\delta$-lactones. The ratio of the natural abundance of the enantiomers of $\delta$-lactones in EVCO was also revealed. Compared with the conventional methods of solvent assisted flavor evaporation (SAFE) and head-space solid-phase micro extraction (HS-SPME), OA-LLE was able to isolate a wide range and large number of volatile compounds from EVCO without leaving oil residues. Therefore, isolating aroma compounds from edible oil based on the oiling-out effect should provide an innovative extraction method.

npj Science of Food (2020)4:18; https://doi.org/10.1038/s41538-020-00079-8

\section{INTRODUCTION}

Edible oil is one of the essential nutrients for human health and is a valuable primary material for many products. Therefore, many kinds of edible oil, e.g., olive oil, coconut oil, safflower oil, and lard, are produced and consumed worldwide. According to the OECDFAO Agricultural Outlook 2019-2028, the global consumption of vegetable oils is over 200 million tonnes/year and is expected to continue to increase ${ }^{1}$ because demand is rising out of proportion to the world population.

It is well known that low molecular weight compounds affect the perception of food as flavor palatability ${ }^{2-4}$. In particular, volatile compounds in foods are a significant factor that affects food intake and preference. To understand the native profile of volatile compounds in edible oils, several methods to extract the volatile compounds have been developed so far.

Head-space solid-phase micro extraction (HS-SPME) introduced by Arthur and Pawliszyn in 1990 has been widely used to extract volatile compounds ${ }^{5}$. HS-SPME is a simple and solvent-free extraction method, and has been used to extract volatile compounds from various kinds of food including edible oils ${ }^{6-9}$. Solvent assisted flavor evaporation (SAFE) introduced by Engel et al. in 1999 has also been used to extract volatile compounds from edible oil ${ }^{10-12}$. SAFE can extract volatile compounds under mild conditions and can separate non-volatiles. In addition, an aromatic extract can be obtained to evaluate aroma characteristics. These methods have greatly contributed to the development of food science and the food industry. However, HS-SPME and SAFE are affected by the matrix effect of oil (triacylglycerols) during extraction processes for oil-enriched samples ${ }^{10,13}$. Some problems remain that need to be resolved, but there are few studies on how to extract volatile compounds from a matrix of oil. Indeed, the latest studies still use the HS-SPME or SAFE methods proposed in the 1990s for extracting volatile compounds from edible oils ${ }^{8,9,12,14,15}$. There have been significant developments in analytical instruments over many years, but extraction methods have become a bottleneck. To encourage and accelerate research on edible oils and fats, an efficient extraction method for volatiles from oil is desired.

Accordingly, we proposed an extraction method for extracting volatile compounds from dark chocolate (fat content, $35.3 \% \mathrm{w} / \mathrm{w}$ ) based on the oiling-out effect ${ }^{16}$. Because the partition coefficient (log Pow) of long-chain triacylglycerols is very high, the triacylglycerols changed the distribution of the hexane and methanol bilayer in liquid-liquid extraction. We found that triacylglycerols are maintained in the hexane layer and the volatile compounds are collected in the methanol layer; this phenomenon was defined by a model study and named the "oiling-out effect". The oiling-out assisted liquid-liquid extraction (OA-LLE) enabled us to extract 54 aroma compounds from only $5 \mathrm{~g}$ of dark chocolate. This method consisted of liquid-liquid extractions, and therefore, there are many advantages, e.g., less susceptible to the matrix effects of triacylglycerols, no heating process, small scale, easy to perform, and no need for expensive equipment. From these results, we hypothesized that OA-LLE can also be used to extract volatile compounds from edible oil. Edible oils consist of mostly triacylglycerols (around 100\%), so edible oils can be regarded as the most difficult sample for extracting volatile compounds due to the strong matrix effect.

Coconut oil, which has a unique aroma, is nowadays widely used around the world. Early studies have shown that the key aroma compounds of coconut meat are $\delta$-lactones, $\delta$-octalactone and $\delta$-decalactone ${ }^{17,18}$. However, there are few studies on volatile compounds in coconut oil ${ }^{19-21}$. Indeed, the enantiomers of $\delta$ lactones contained in coconut oil have not been reported. Data on the enantiomer ratio of aroma compounds is important in helping to understand the aroma characteristics and the authenticity control of flavors ${ }^{22}$. However, the volatile composition of coconut oil is poorly understood due to a strong matrix effect.

In this study, OA-LLE was used to extract volatile compounds from only $5 \mathrm{~g}$ of extra virgin coconut oil (EVCO) as a typical model, and we evaluated the extraction efficiency of OA-LLE compared with HS-SPME and SAFE. In addition, the enantiomers of

\footnotetext{
${ }^{1}$ Institute of Health Sciences, Ezaki Glico Co., Ltd., 4-6-5 Utajima, Nishiyodogawa-ku, Osaka 555-8502, Japan. ${ }^{2}$ The United Graduate School of Agricultural Sciences, Ehime University, 3-5-7 Tarumi, Matsuyama-shi, Ehime 790-8566, Japan. ${ }^{3}$ The Graduate School of Agriculture, Kagawa University, 2393 Ikenobe, Miki-cho, Kagawa 761-0795, Japan.

email: daisuke.suzuki@glico.com
} 


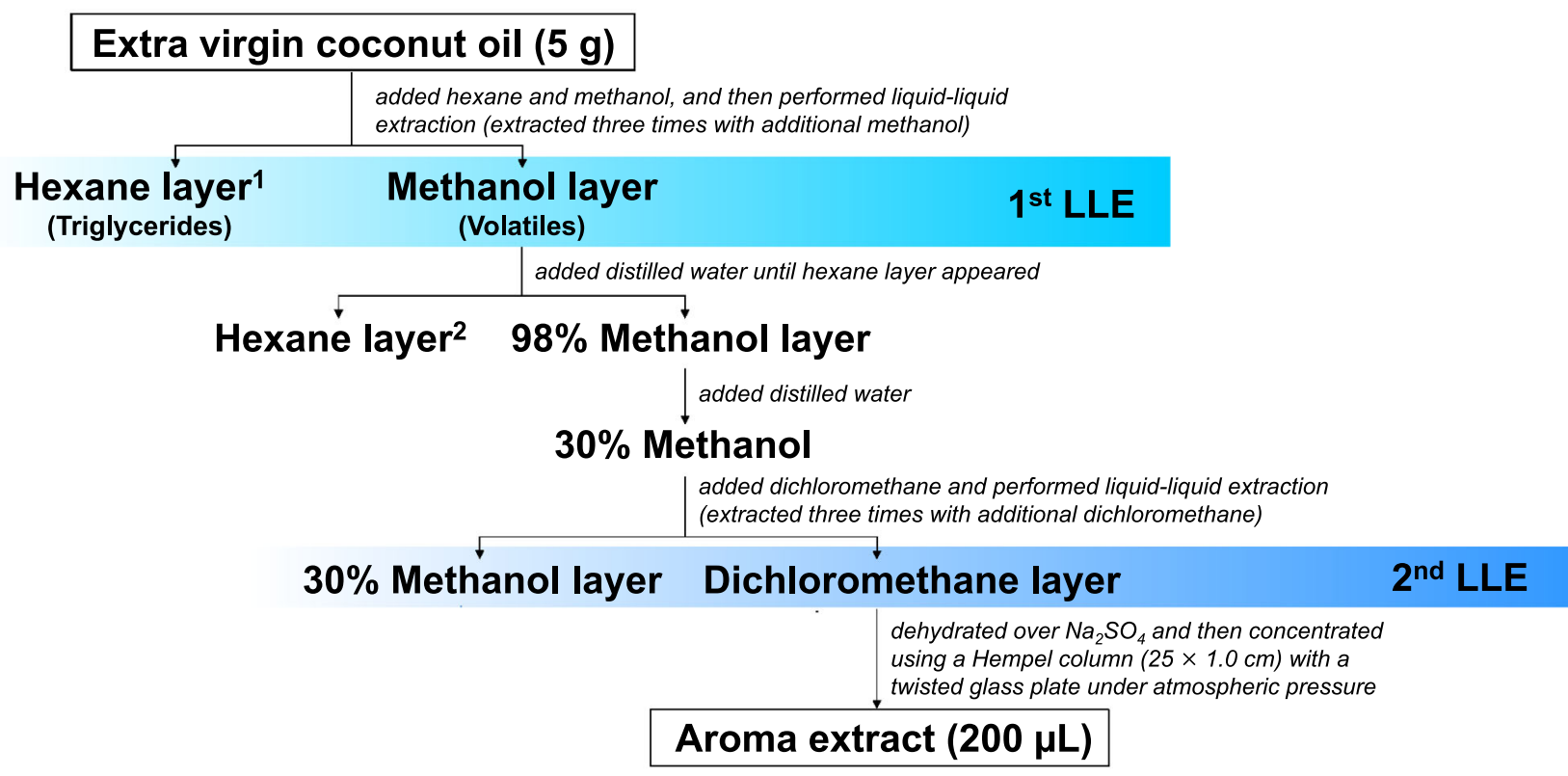

Fig. 1 Procedure of the oiling-out assisted liquid-liquid extraction (OA-LLE) for extra virgin coconut oil (EVCO). OA-LLE COnsists of two small-scale liquid-liquid extractions.

$\delta$-lactones in EVCO were analyzed by gas chromatography-mass spectrometry (GC-MS) equipped with an enantioselective column, the results of which were reported. The method described in this paper should prove to be valuable in determining the volatile composition of edible oils, and should contribute to promoting research in food science as well as its associated industry.

\section{RESULTS}

Extraction of volatile compounds in EVCO using OA-LLE

Liquid-liquid extraction is a fundamental and important separation technique for a wide range of applications in scientific research and industries. Figure 1 presents the procedure of OA-LLE for EVCO. OA-LLE consists of two liquid-liquid extractions, hexane/ methanol and 30\% methanol solution/dichloromethane bilayers. At the first liquid-liquid extraction, the oil content in "hexane layer" ${ }^{1 \prime}$ was $20.0 \%(\mathrm{w} / \mathrm{v})$. From our previous study, it was considered that the oiling-out effect occurred ${ }^{16}$. After the OA-

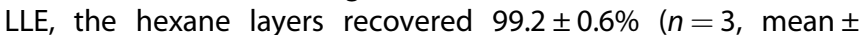
standard deviation (SD), $w / w$ ) of the oil, indicating most of triacylglycerol was maintained in the hexane layer. To confirm the removed layers ("hexane layer", "hexane layer", and "30\% methanol layer") were odorless, three researchers sniffed a mouillette (smelling strip) dipped in these layers and sensorily judged that there was no odor in "hexane layer" ${ }^{1 "}$ and " $30 \%$ methanol layer". There was a very slight odor in "hexane layer", perhaps because it did not contain enough oil for the oiling-out effect to occur. In contrast, the extract (concentrate of the "dichloromethane layer") had a strong coconut-like aroma, and the typical aroma of coconut was recovered. These results indicate that most of the volatile compounds in EVCO were extracted by using OA-LLE. The extract was used for further analysis.

\section{Volatile compounds in EVCO}

The GC-MS chromatograms and volatile compounds from OA-LLE, SAFE, and HS-SPME are shown in Fig. 2 and Table 1, respectively. In all, 50 volatile compounds were identified in EVCO using the three methods. With OA-LLE, 44 volatile compounds, comprising 8 acids, 13 alcohols, 3 aldehydes, 4 esters, 3 hydrocarbons, 7 ketones, and 6 lactones, were identified. Most of the volatile compounds detected in EVCO, such as nonanal and decanoic acid, were derived from triacylglycerols. $\delta$-Lactones, $\delta$-hexalactone $(\delta$ $\left.C_{6}\right)$, $\delta$-octalactone $\left(\delta-C_{8}\right), \delta$-decalactone $\left(\delta-C_{10}\right), \delta$-dodecalactone $\left(\delta-C_{12}\right), \delta$-tetradecalactone $\left(\delta-C_{14}\right)$, and $\delta$-hexadecalactone $\left(\delta-C_{16}\right)$, were the major component of the volatiles in EVCO, and the content of these $\delta$-lactones in the extract was $53.5 \pm 6.2,173.0 \pm$ $14.6,353.0 \pm 17.5,277.6 \pm 10.7,13.6 \pm 0.9$, and $2.1 \pm 0.6 \mu \mathrm{g} / 200 \mu \mathrm{L}$ $(n=3$, mean $\pm S D)$, respectively. A large amount of $\delta$-lactones was extracted. Applying available thresholds in oil or water, the odor activity value (OAV) (also odor unit) was calculated. The potential odorants $(\mathrm{OAV}>1)$ detected in the OA-LLE extract are listed in Table 2. In total, 14 potential odorants were found. $\delta$-Lactones have a chiral center in their compounds, so these compounds have enantiomers. As shown in Fig. 3 and Table 3, the predominance of $(\mathrm{R})$-enantiomers decreased with an increasing lactone chain length by $\delta-C_{12} . \delta-C_{14}$ and $\delta-C_{16}$ (R)-enantiomers increased up to 72.1 and $95.9 \%$, respectively.

Comparison of extraction efficiency among the methods

The log Pow value of the volatile compounds isolated by each method was plotted and calculated statistically (Fig. 4). A wide range of volatile compounds from log Pow - 0.3 (2,5-hexanedione) to log Pow 8.4 (1-octadecanol) were isolated from the triacylglycerols using OA-LLE. Using SAFE, 17 volatile compounds were extracted. The range of log Pow values of the volatile compounds was from 1.0 ( $\delta$-hexalactone) to 5.6 (ethyl dodecanoate and 2-tridecanol). Comparing the results of OA-LLE and SAFE, the variances in log Pow values were 4.26 and 1.67 , which were statistically different $(p<0.05)$. Using HS-SPME, 20 of the volatile compounds in EVCO were identified with log Pow from -0.2 (acetic acid) to log Pow 6.1 (dodecane). The variance for HS-SPME was 3.11, and the variances of OA-LLE and HS-SPME were not statistically different $(p=0.4350)$. The variances of SAFE and HSSPME were also not statistically different $(p=0.2076)$.

The total amounts of the aroma compounds in the extracts from OA-LLE and SAFE were 1,066.1 and $99.5 \mu \mathrm{g} / 200 \mu \mathrm{L}$, respectively. Using OA-LLE, we were able to extract over 10-fold the amount of aroma compounds from EVCO compared with SAFE. $\delta$-Lactones, $\delta-C_{6}$ to $\delta-C_{12}$, were detected, and the content of these $\delta$-lactones in the extract from SAFE were $16.0 \pm 2.2,40.9 \pm$ $1.8, \quad 25.5 \pm 5.6$, and $4.1 \pm 1.5 \mu \mathrm{g} / 200 \mu \mathrm{L} \quad(n=3, \quad$ mean $\pm S D)$, 
a

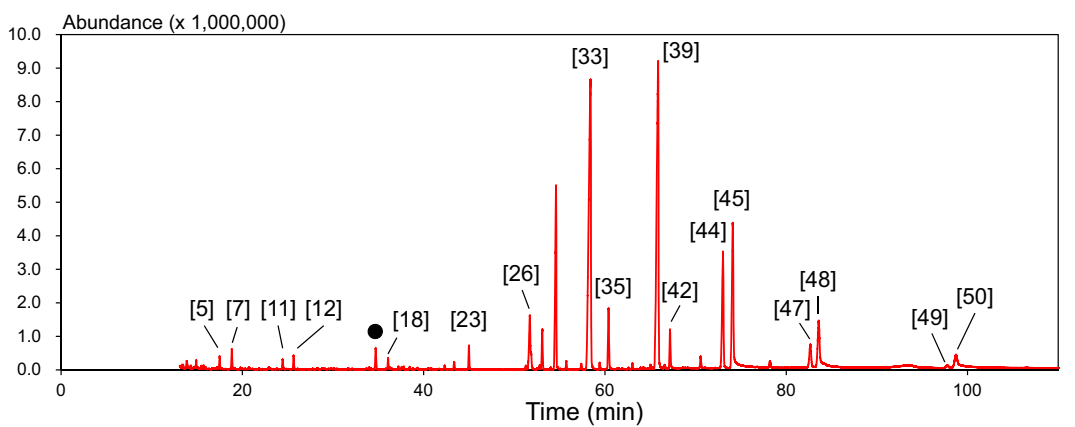

b

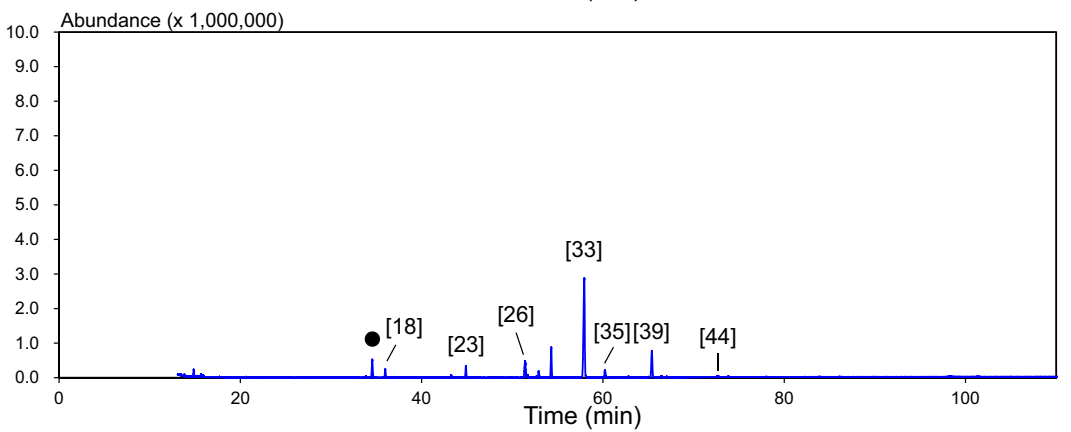

C

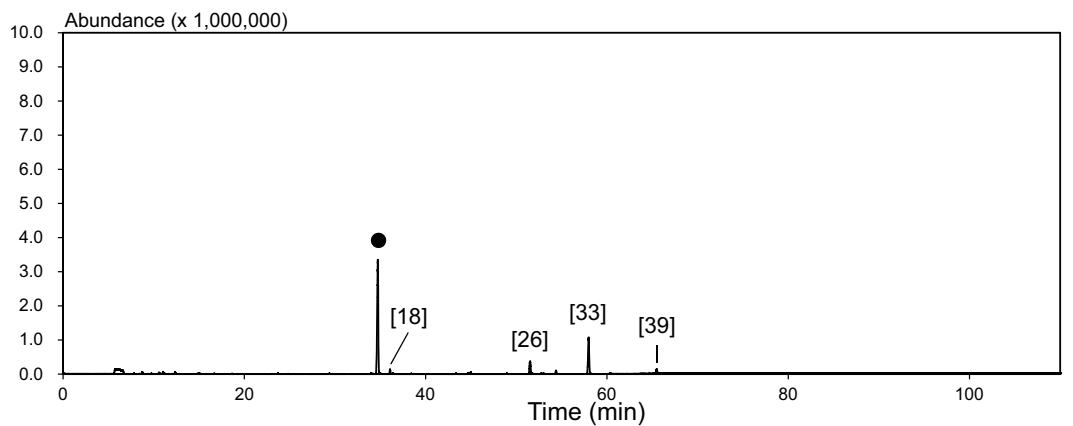

Fig. 2 GC-MS chromatograms of the EVCO extracts from each extraction method. The chromatograms of OA-LLE, solvent assisted flavor evaporation (SAFE), and head-space solid-phase micro extraction (HS-SPME) are shown in a-c, respectively. Numbering refers to the volatile compounds listed in Table 1. Filled black circles $(\cdot)$ indicate internal standard peaks (cyclohexanol).

respectively. Compared with the result from OA-LLE, the amount of $\delta$-lactones in the extract from SAFE was much lower, and $\delta-C_{14}$ and $\delta-C_{16}$ were not detected in the SAFE extract. There were no specific compounds found by SAFE but not by OA-LLE. During the SAFE distillation, EVCO accumulated at the high vacuum stopcock and flash distillation may not have worked well (Supplementary Fig. 1).

The sums of the peak abundances of the GC-MS chromatograms from OA-LLE, SAFE, and HS-SPME were 429,939.8, 51,622.8, and $17,291.9$, respectively. The total abundance of HS-SPME was the lowest due to the matrix effect. The specific compounds found by HS-SPME but not by OA-LLE were as follows: 1 alcohol (ethanol), 1 aldehyde (hexanal), 1 ketone (2-heptanone), and 3 hydrocarbons (octane, decane, and dodecane). The extraction efficiency of the volatile compounds from EVCO was dramatically improved using OA-LLE.

Similarity and intensity test of the aromatic extracts from OA-LLE and SAFE

Similarity and intensity of EVCO aroma perceived by the panelists for the extracts obtained by OA-LLE and SAFE were tested. The mean similarity scores of the aromatic extracts obtained from OALLE and SAFE on the smelling strips were 71.4 \pm 7.3 and $37.4 \pm$ $6.6 \mathrm{~mm}(n=5$, mean \pm standard error (SE)), respectively, and were statistically different $(p<0.05)$. The mean intensity scores of the aromatic extracts obtained by OA-LLE and SAFE on the smelling strips were $67.4 \pm 11.9$ and $75.0 \pm 7.0 \mathrm{~mm} \quad(n=5$, mean $\pm \mathrm{SE})$, respectively, and there was no statistical difference $(p=0.5650)$. These results indicate that the OA-LLE extract had a similar aroma composition to the reference sample (EVCO).

\section{DISCUSSION}

To understand the native profile of volatile compounds in edible oil, we need to overcome the strong matrix effect of oil. Solvent extraction, which is the most widely used method for extracting volatiles, is less susceptible to matrix effects. However, solvent extraction is unsuitable for extracting volatile compounds from oil because volatile compounds and oil are extracted simultaneously. A large amount of oil in the isolate makes it difficult to concentrate volatile compounds ${ }^{16}$. OA-LLE proposed in this paper consists of liquid-liquid extractions, which is less affected by the matrix effect of oil because of solvent-based extraction. The oilingout effect for EVCO is illustrated in Fig. 5. The oiling-out effect was defined in our previous study ${ }^{16}$.

In brief, we focused on the partition coefficient of oil (middleand long-chain triacylglycerols) and demonstrated that triacylglycerols affected the distribution of the hexane/methanol bilayer and the equilibrium distribution of aroma compounds when performing liquid-liquid extraction. The partition coefficient of medium- and long-chain triacylglycerols is extremely high (log Pow value $>10$ ). According to the PubChem database, the log Pow 


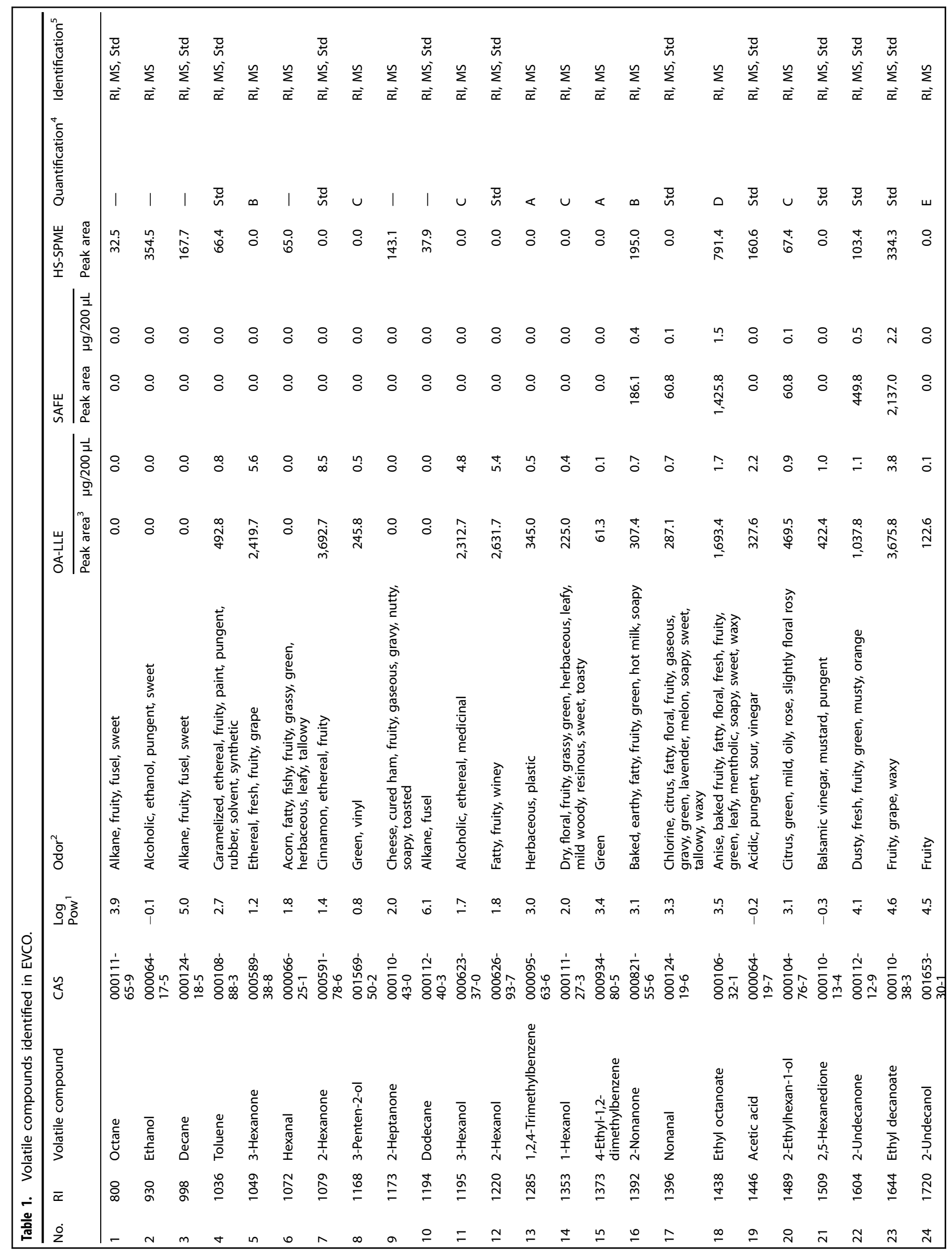




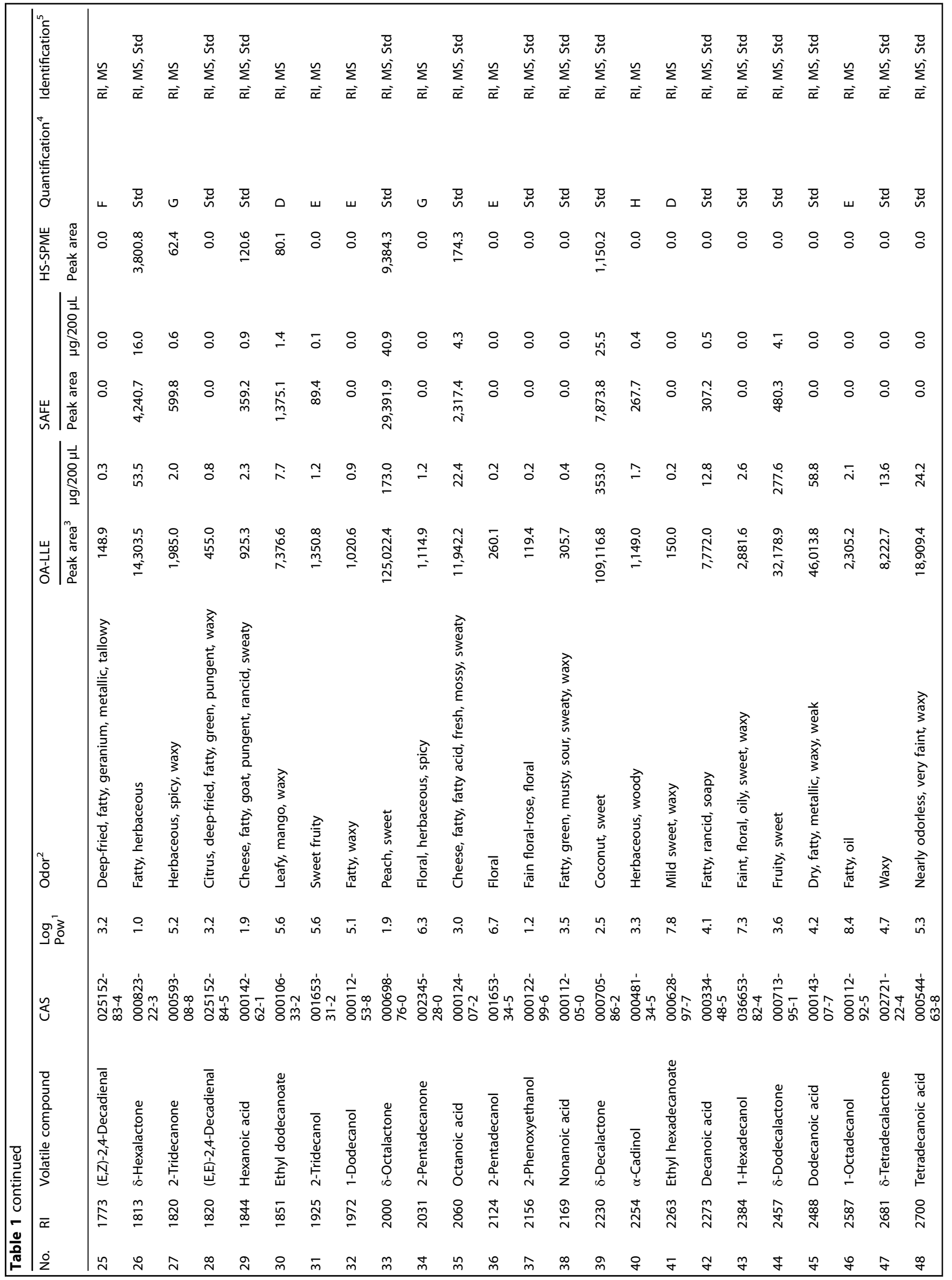


Table 2. $\operatorname{OAV}^{1}(>1)$ of aroma compounds in the EVCO extract obtained by OA-LLE.

\begin{tabular}{|c|c|c|}
\hline Compound & Odor $^{2}$ & $O A V^{1}$ \\
\hline$\delta$-Dodecalactone & Fruity, sweet & 462.6 \\
\hline$\delta$-Decalactone & Coconut, sweet & 176.5 \\
\hline 2-Hexanone & Cinnamon, ethereal, fruity & $\underline{21.1}$ \\
\hline Ethyl decanoate & Fruity, grape, waxy & 19.2 \\
\hline 3-Hexanone & Ethereal, fresh, fruity, grape & 18.7 \\
\hline$\delta$-Octalactone & Peach, sweet & 13.9 \\
\hline (E,Z)-2,4-Decadienal & $\begin{array}{l}\text { Deep-fried, fatty, geranium, metallic, } \\
\text { tallowy }\end{array}$ & 7.3 \\
\hline Ethyl octanoate & $\begin{array}{l}\text { Anise, baked fruity, fatty, floral, fresh, } \\
\text { fruity, green, leafy, mentholic, soapy, } \\
\text { sweet, waxy }\end{array}$ & $\underline{5.0}$ \\
\hline 2-Hexanol & Fatty, fruity, winey & $\underline{4.3}$ \\
\hline 2-Nonanone & $\begin{array}{l}\text { Baked, earthy, fatty, fruity, green, hot } \\
\text { milk, soapy }\end{array}$ & 1.4 \\
\hline Acetic acid & Acidic, pungent, sour, vinegar & 1.4 \\
\hline 1-Dodecanol & Fatty, waxy & 1.4 \\
\hline 3-Hexanol & Alcoholic, ethereal, medicinal & $\underline{1.2}$ \\
\hline 2-Undecanol & Fruity & 1.1 \\
\hline
\end{tabular}

'Odor activity value. OAV was calculated as follows: the concentration of aroma compounds $(\mu \mathrm{g} / 200 \mu \mathrm{L})$ shown in Table 1 was regarded as the concentration of aroma compounds in $5 \mathrm{~g}$ of EVCO and was converted to $\mathrm{mg} / \mathrm{kg}$ by multiplying by 200 . The converted concentration of aroma compounds/odor threshold in oil or water from AroChemBase. The underlines indicate that the OAVs were calculated using a threshold in water.

${ }^{2}$ Odor descriptions were obtained from AroChemBase.

value of trilaurin, the most abundant triacylglycerol in coconut oil, is 15.6. This phenomenon is similar to the "salting-out effect" of chemicals in aqueous solution by adding sodium chloride. As with the salting-out effect, adding low polarity compounds immiscible with water, such as oil, changes the polarity of the hexane layer and pushes out relatively hydrophilic compounds into the methanol layer. In addition, there is no heating process in OALLE. Heating should be avoided to generate artifact compounds during the extraction procedure. In this study, we applied OA-LLE to EVCO as a typical model to propose a fresh approach to extracting volatile compounds from edible oil. As a result, a wide variety and large number of volatile compounds could be isolated from only $5 \mathrm{~g}$ of EVCO. In addition, the aroma characteristic of the OA-LLE extract was similar to the reference sample (EVCO).

The main component of the volatiles in EVCO was $\delta$-lactones, which corresponded to the results of previous studies ${ }^{19-21}$. The content of $\delta$-lactones in EVCO was also similar to previous studies, indicating most of the volatile compounds were extracted from EVCO with OA-LLE (Supplementary Table 1$)^{19,21,23}$. In this study, $\delta$ $C_{16}$ was detected in EVCO. Because $\delta-C_{16}$ has a high log Pow value, it is considered that these compounds were concentrated in EVCO from coconut meat during the production processes of EVCO. $\delta-C_{16}$ contributes to the "buttery" sensation of food, but it may be difficult to evaluate GC-olfactometry and OAV due to the high boiling point ${ }^{24}$.

From our results, it was found that there are many semi-volatile compounds and compounds with high affinity for oil other than $\delta$ lactones in EVCO. Edible oils such as olive oil and coconut oil are consumed as food in general. To understand the aroma and flavor characteristics of edible oils, we should focus on not only volatile compounds but also semi-volatile compounds and compounds with a high affinity for oil. To the best of our knowledge, 23 of the aroma compounds, comprising 2 acids (nonanoic acid and 
a

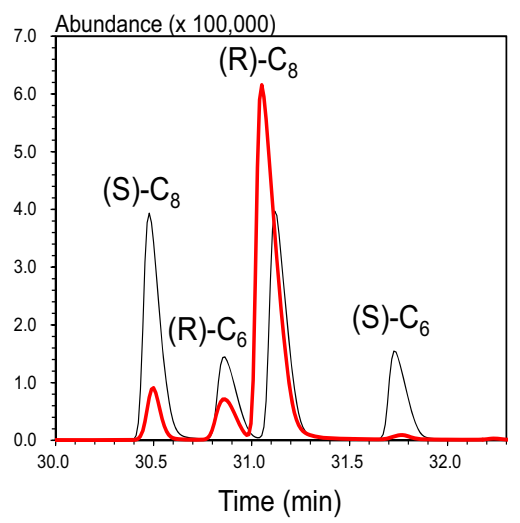

d

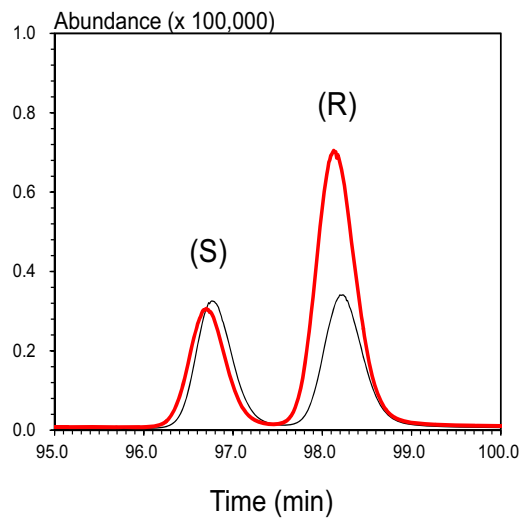

b

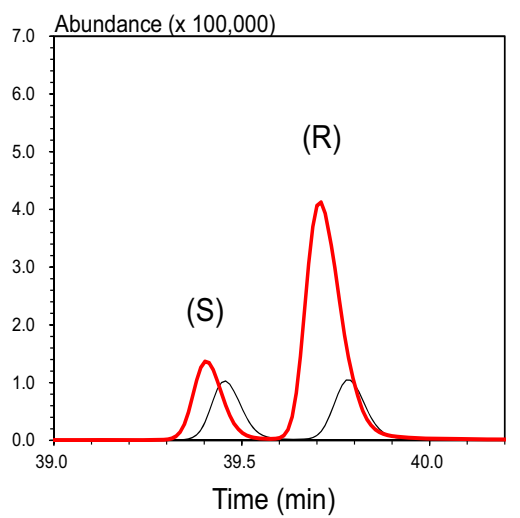

e

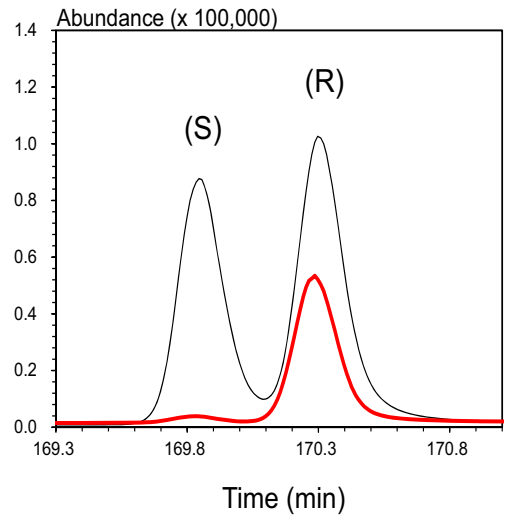

C

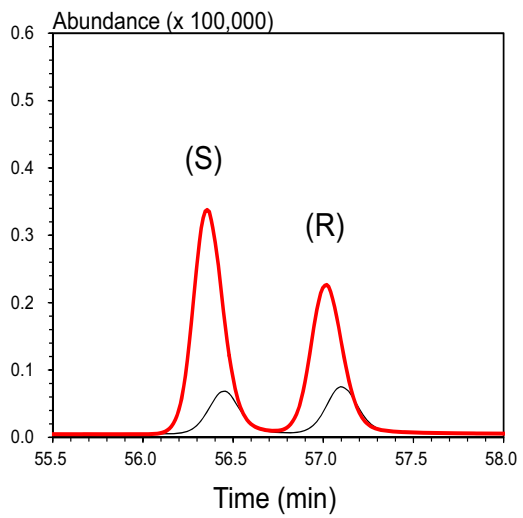

Fig. 3 Enantioselective GC-MS chromatograms of $\boldsymbol{\delta}$-lactones. The chromatograms of the OA-LLE extract are drawn with a red and bold line, and a black line denote those of the standard solutions. The peaks of $\delta$-hexalactone, $\delta$-octalactone, $\delta$-decalactone, $\delta$-dodecalactone, $\delta$ tetradecalactone, and $\delta$-hexadecalactone are shown in a, a-e, respectively. Each \% area of (R)- and (S)-enantiomers is shown in Table 3.

Table 3. \% Area of the (R)- and (S)- $\delta$-lactone enantiomers in the EVCO extract obtained by OA-LLE.

\begin{tabular}{llr}
\hline Lactone & & \% Area \\
\hline$\delta$-Hexalactone & $(\mathrm{R})$ & $89.1 \pm 0.1$ \\
& $(\mathrm{~S})$ & $10.9 \pm 0.1$ \\
$\delta$-Octalactone & $(\mathrm{R})$ & $90.8 \pm 0.1$ \\
& $(\mathrm{~S})$ & $9.2 \pm 0.1$ \\
$\delta$-Decalactone & $(\mathrm{R})$ & $78.3 \pm 0.1$ \\
& $(\mathrm{~S})$ & $21.7 \pm 0.1$ \\
$\delta$-Dodecalactone & $(\mathrm{R})$ & $41.3 \pm 0.1$ \\
& $(\mathrm{~S})$ & $58.7 \pm 0.1$ \\
$\delta$-Tetradecalactone & $(\mathrm{R})$ & $72.1 \pm 0.4$ \\
$\delta$-Hexadecalactone & $(\mathrm{S})$ & $27.9 \pm 0.4$ \\
& $(\mathrm{R})$ & $95.9 \pm 0.1$ \\
The data represent the mean $\pm \mathrm{SD}(n=3)$. & \\
\hline
\end{tabular}

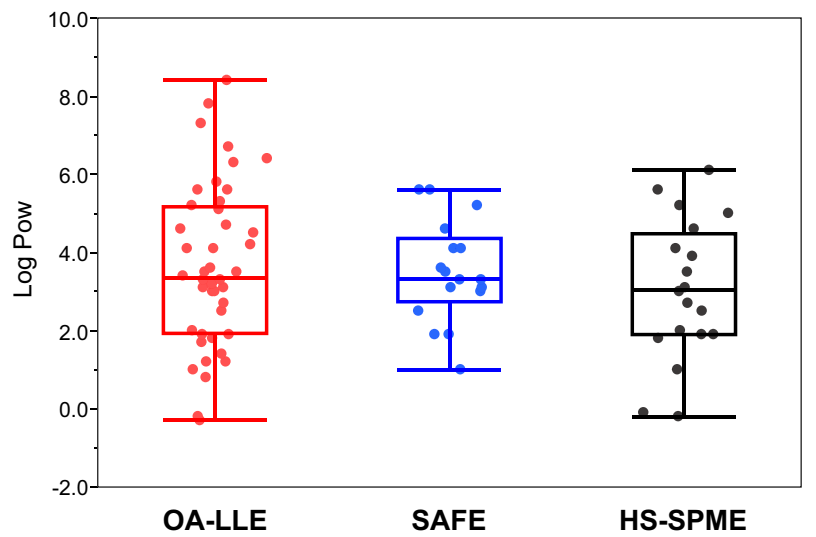

Fig. 4 Box plots of the log Pow values for the volatile compounds extracted with each method. The distribution of the data is summarized in box plots and the log Pow values for each aroma compound are plotted. The center line in the box plots is the median, the box denotes the interquartile range (IQR), and whiskers are drawn to the furthest point within $1.5 \times \mathrm{IQR}$ from the box. The log Pow values were quoted from PubChem database (last access date: 27 May 2020). The number of volatile compounds of OA-LLE, SAFE, and HS-SPME was 44, 17, and 20, respectively. 


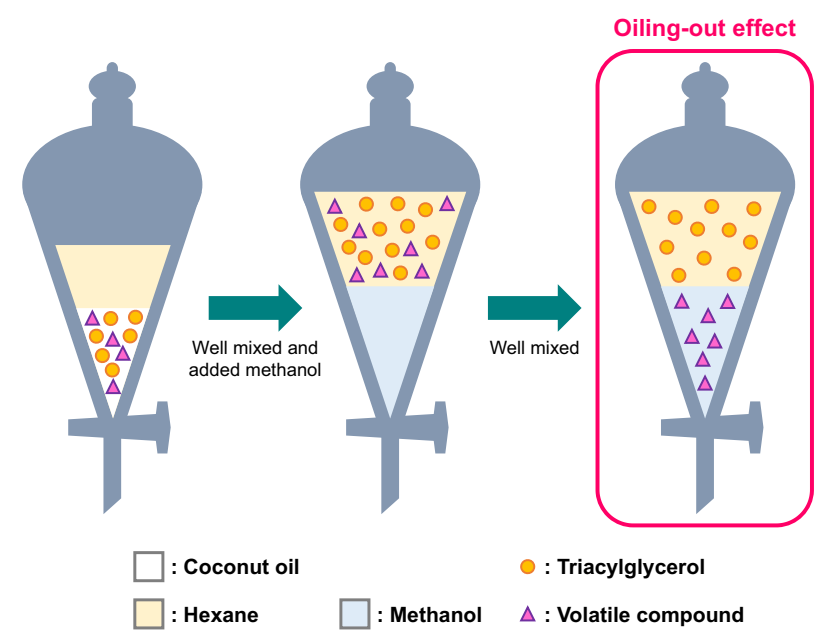

Fig. 5 Image of the oiling-out effect in the extraction procedure of EVCO. This liquid-liquid extraction is the first step of OA-LLE. The hexane layer maintained triacylglycerols and pushed out relatively hydrophilic compounds (volatile compounds) into the methanol layer due to the oiling-out effect.

hexadecanoic acid), 13 alcohols (3-penten-2-ol, 3-hexanol, 2hexanol, 1-hexanol, 2-ethylhexan-1-ol, 2-undecanol, 2-tridecanol, 1-dodecanol, 2-pentadecanol, 2-phenoxyethanol, a-cadinol, 1hexadecanol, and 1-octadecanol), 2 aldehydes ((E,Z)-2,4-decadienal and (E,E)-2,4-decadienal), 3 hydrocarbons (toluene, 1,2,4trimethylbenzene, and 4-ethyl-1,2-dimethylbenzene), 2 ketones (3-hexanone and 2,5-hexanedione), and 1 lactone ( $\delta$-hexadecalactone), were newly found in coconut oil using OA-LLE ${ }^{19-21}$. The alcohols have a fruity or floral aroma and might contribute to the EVCO aroma. Moreover, 14 of the potential odorants were found by OAV. As for the aroma component in coconut oil, compounds other than $\delta$-lactones have not been focused on so far.

We also revealed the enantiomers of $\delta$-lactone in EVCO, as shown in Fig. 3 and Table 3. Because enantiomers have different aroma characteristics in general, the data on the enantiomeric ratio are important in helping to understand the native aroma ${ }^{25}$. The $\%$ area of (R)- and (S)-enantiomers of $\delta-C_{14}$ and $\delta-C_{16}$ in the coconut product were revealed. The $\delta-C_{6}$ to $\delta-C_{12}$ of $\%$ area were similar to previous studies ${ }^{26}$. Notably, the predominance of $(R)-$ enantiomers decreased with an increasing lactone chain length until $\delta-C_{12}$, but increased at $\delta-C_{14}$ and $\delta-C_{16}$ (Table 3 ). Lauric acid is the most abundant fatty acid in coconut oil, which might be related to the enantiomeric ratio.

There are four common methods for producing coconut oils, namely expelling, centrifugation, and fermentation with and without heating ${ }^{27}$. In addition, there are many types of processing, such as bleached, resulting in diversified flavors ${ }^{28}$. The application of OA-LLE to EVCO revealed aroma components other than $\delta$ lactones. The minor aroma activity compounds may characterize the variety of coconut oils. Traditionally, coconut oils have been used not only for foods but also for non-food applications ${ }^{29,30}$. Thus, wider research on the volatile composition of coconut oils should be conducted. To identify key aroma compounds in edible oil, applying aroma extract dilution analysis (AEDA) or charm analysis for the extract obtained by OA-LLE would be helpful ${ }^{31,32}$. The semi-volatiles can be subjected to sensory evaluation by adding a standard compound (food-grade is desirable) to the substrate. We can relate aroma compounds in the extract from OA-LLE with sensory attributes by means of pattern recognition techniques that use multivariate statistical analysis such as partial least square (PLS) algorithms as in previous studies ${ }^{33,34}$.

The sensory panelists in this study judged that the SAFE extract from EVCO was not similar to the reference; only 17 volatile compounds were identified. To the best our knowledge, no study has ever applied SAFE to coconut oil. In this experiment, it was difficult to use SAFE to extract the volatile compounds from EVCO as shown in Supplementary Fig. 1. Because the melting point of EVCO is high, it is considered that EVCO solidified because of the heat of vaporization generated by flash distillation. Applying SAFE to other oils that have a high melting point, such as palm oil and lard, might also be difficult. HS-SPME is also affected by the matrix effect of oil, resulting in the lowest total abundance of peak areas. On the other hand, HS-SPME is a solventless method, so there was no solvent peak in the chromatogram, resulting in 20 volatile compounds being extracted. These results indicate that HS-SPME is suitable for analyzing volatile compounds with a low boiling point. Our previous study showed that applying OA-LLE to $5 \mathrm{~g}$ of dark chocolate (fat content, $35.3 \% \mathrm{w} / \mathrm{w}$ ) was successful in extracting volatile compounds. With an oil content of $100 \%$, EVCO can be regarded as a sample having the strongest matrix effect. Our results here indicate that OA-LLE is a powerful tool for understanding the aroma profiles of edible oils. Indeed, OA-LLE can be applied to other edible oils (olive oil and beef tallow) and was successful in extracting volatile compounds as shown in Supplementary Fig. 2.

Applying OA-LLE to edible oil and oil-enriched food indicates the potential for other studies. Lukić et al. reported on 256 volatile compounds in olive oils using comprehensive two-dimensional gas chromatography with time-of-flight mass spectrometry (GC $\times$ GC-TOF-MS) combined with conventional mono-dimensional GC$M^{8}$. They extracted volatile compounds in olive oil using HSSPME. There have been significant developments in analytical instruments over many years, so combining OA-LLE with highperformance analytical instruments may give a deeper insight into the volatile composition of edible oils. The resulting data should help to reveal geographical or variety differences in oil crops as in previous studies ${ }^{35,36}$. Elucidating the mechanisms of triacylglycerol oxidation is also valuable for food science and the food industry. Recently, a method for analyzing triacylglycerol oxidation in detail has been proposed ${ }^{37}$. The data on the native profile of volatile components derived from triacylglycerols may be useful in elucidating the triacylglycerol oxidation mechanisms in edible oils.

For sustainable supply of foods and for reasons of health, controlling food intake and weight gain have become significant issues. Therefore, understanding the mechanisms of oil and fat perception is desired. Lee et al. reported on the involvement of cluster of differentiation 36 (CD36) on the olfactory epithelium in mice in the perception of oleic aldehyde ${ }^{38}$. In addition, the assay system of human CD36 (peptide mimic) was developed to discover additional potential ligands of CD36 $6^{39,40}$. Applying OALLE for edible oils should provide candidates for potential ligands of CD36, and the findings may help to understand the mechanisms of food perception.

In this study, we demonstrated an effective method for extracting volatile compounds from edible oils. As a result, the volatile list, aroma characteristics, data on potential odorants, and the enantiomeric ratio of $\delta$-lactones were obtained from only $5 \mathrm{~g}$ of EVCO. Moreover, OA-LLE is simple, rapid, cost-effective, and does not require a heating process. This method is applicable to a wide variety of edible oils and oil- or fat-enriched foods and should provide a new insight into their aroma profiles.

\section{METHODS}

Reagents and samples

All the solvents and reagents used were commercially available. Hexane $(96.0+\%)$ and methanol $(99.8 \%)$ were purchased from Kishida Chemicals Co., Ltd. (Osaka, Japan). Acetic acid (>99.7\%), dichloromethane (99.5\%), 2,4decadienal $(90.0 \%+)$, hexanoic acid $(>99.0 \%)$, nonanal $(>95.0 \%)$, nonanoic acid $(90.0 \%+)$, tetradecanoic acid $(>98.0 \%)$, and toluene $(>99.5 \%)$ were purchased from FUJIFILM Wako Pure Chemical Corporation (Osaka, Japan). 
$\delta$-Hexanolactone (>99.0\%), $\delta$-octanolactone $(>98.0 \%), \delta$-decanolactone $(>97.0 \%), \delta$-dodecanolactone $(>98.0 \%), \delta$-tetradecanolactone $(>98.0 \%)$, ethyl decanoate $(>98.0 \%)$, 1-hexadecanol $(>98.0 \%)$, 2-hexanol $(>98.0 \%), 2$ hexanone $(>98.0 \%)$, octanoic acid $(>98.0 \%)$, 2-phenoxyethanol $(>98.5 \%)$, and 2-undecanone (>98.0\%) were obtained from Tokyo Chemical Industry Co., Ltd. (Tokyo, Japan). Decanoic acid (>99.0\%) and dodecanoic acid (>99.0\%) were purchased from Sigma-Aldrich (St. Louis, MO, USA). Hexadecanoic acid was acquired from Nacalai Tesque, Inc. (Kyoto, Japan). $\delta$-Hexadecanolactone $(>98.0 \%)$ was purchased from Soda Aromatic Co., Ltd. (Tokyo, Japan). 2,5-Hexanedione was obtained from Kanto Chemical Co., Inc. (Tokyo, Japan). Alkane mixed solution $\left(C_{7}-C_{33}\right.$, in hexane) was obtained from Hayashi Pure Chemical Ind., Ltd. (Osaka, Japan). Extra virgin coconut oil (EVCO) made in Thailand was purchased from a local market in Japan and stored at $-20^{\circ} \mathrm{C}$ until used. The EVCO was made using a centrifuge without heating, and the production date was December 2018.

\section{Extraction of volatile compounds in EVCO}

Oiling-out assisted liquid-liquid extraction (OA-LLE). The procedure for extracting volatile compounds in EVCO is shown in Fig. 1. First, $5 \mathrm{~g}$ of EVCO and $20 \mathrm{~mL}$ of hexane were charged into a separatory funnel and then well mixed. After that, $10 \mathrm{~mL}$ of methanol were charged into the separatory funnel and liquid-liquid extraction was performed. The lower methanol layer containing volatiles was recovered, and then the remaining volatile compounds in the upper oil-rich hexane layer were extracted three times with methanol $(5.0,2.5$, and $2.5 \mathrm{~mL}$, respectively). Distilled water $(531 \mu \mathrm{L})$ was added to the combined mixture of methanol layers $(26.0 \mathrm{~mL}$, saturated with hexane) until insoluble hexane appeared. After that, the separated hexane layer was removed. Next, distilled water $(60.1 \mathrm{~mL})$ was added to the aqueous methanol solution to prepare a $30 \%$ aqueous methanol solution. Finally, volatile compounds were extracted four times with dichloromethane $(28.9,14.4,14.4$, and $14.4 \mathrm{~mL})$ from the $30 \%$ aqueous methanol layer. The dichloromethane layer was treated with $10 \mathrm{~g}$ of anhydrous sodium sulfate to remove the water (overnight at $-20^{\circ} \mathrm{C}$ ). The dehydrated dichloromethane layer was concentrated to $200 \mu \mathrm{L}$ using a Hempel column $(25 \times 1.0 \mathrm{~cm})$ with a twisted glass plate under atmospheric pressure (ca. $43^{\circ} \mathrm{C}$ ). To calculate the recovery of oil, the removed hexane layers were evaporated and dried using a vacuum drying system for $2 \mathrm{~h}$ at room temperature. A $2 \mu \mathrm{L}$ aliquot of the EVCO extract was used for GC-MS analysis. The extraction was performed in triplicate.

Solvent assisted flavor evaporation (SAFE). The method was in accordance with Peres et al. with a minor modification ${ }^{11}$. First, $5 \mathrm{~g}$ of EVCO and $25 \mathrm{~mL}$ of dichloromethane were placed into a flat-bottom flask with a cap and well mixed. The sample solution was charged into a SAFE apparatus with an oil diffusion pump $\left(10^{-3} \mathrm{~Pa}\right)^{10}$. The gentle distillation of volatiles under a high vacuum distillation system thermostated at $40^{\circ} \mathrm{C}$ resulted in the volatiles in dichloromethane. The isolate was dried with $10 \mathrm{~g}$ of anhydrous sodium sulfate (overnight at $-20^{\circ} \mathrm{C}$ ) and concentrated to $200 \mu \mathrm{L}$ using a Hempel column $(25 \times 1.0 \mathrm{~cm})$ with a twisted glass plate under atmospheric pressure (ca. $43^{\circ} \mathrm{C}$ ). A $2 \mu \mathrm{L}$ aliquot of the EVCO extract was used for GC-MS analysis. The extraction was performed in triplicate.

Head-space solid-phase micro extraction (HS-SPME). HS-SPME was conducted using the method described by Santos et al. with a minor modification $^{21}$. In brief, $5 \mathrm{~g}$ of EVCO was placed into a $20-\mathrm{mL}$ glass serum vial and $1 \mu \mathrm{L}$ of cyclohexanol was added as an internal standard. The vial was subsequently screw-capped with a laminated Teflon-silicone disc. HSSPME was performed with a 50/30 $\mu \mathrm{m}$ divinylbenzene/carboxen/polydimethylsiloxane (DVB/CAR/PDMS) fiber mounted on a SPME manual holder assembly (Supelco, Inc., Bellefonte, PA, USA). The vial containing EVCO was placed in a $40^{\circ} \mathrm{C}$ water bath and maintained for 5 min with stirring. The needle of the SPME device was then inserted into the vial through the septum, and the plunger of the SPME apparatus was pushed down to expose the SPME fiber to the vial head space. After $30 \mathrm{~min}$ exposure, the fiber was retracted into the needle assembly, removed from the vial, and then introduced into a preheated GC-MS injector port for analyte desorption at $230^{\circ} \mathrm{C}$ for $5 \mathrm{~min}$. HS-SPME analysis was performed in triplicate.

\section{Gas chromatography-mass spectrometry (GC-MS)}

The volatile compounds were isolated and identified using a $7890 \mathrm{~A} \mathrm{GC}$ System gas chromatograph (Agilent Technologies, Inc., Santa Clara, CA, USA) coupled with a 5975 C inert XL MSD mass spectrometer detector
(Agilent Technologies, Inc.). The GC-MS system was equipped with a capillary column DB-WAX Ultra Inert $(30 \mathrm{~m} \times 0.25 \mathrm{~mm}$ i.d., $0.25 \mu \mathrm{m}$ film thickness; Agilent Technologies, Inc.). The carrier gas was helium (99.995\%) and the column head pressure was set at $108 \mathrm{kPa}$. The oven temperature was set at $30^{\circ} \mathrm{C}$ for $2 \mathrm{~min}$, increased to $230^{\circ} \mathrm{C}$ at $3{ }^{\circ} \mathrm{C} \mathrm{min}{ }^{-1}$, and finally maintained at $230^{\circ} \mathrm{C}$ for $45 \mathrm{~min}$. The splitless injection mode was at $230^{\circ} \mathrm{C}$. Mass spectra in the electron impact mode (EI) were generated at $70 \mathrm{eV}$ and the MSD transfer line temperature was set to $230^{\circ} \mathrm{C}$. The mass scan range was 30 to $400 \mathrm{~m} / \mathrm{z}$.

\section{Identification and quantification of volatiles}

To identify the volatile compounds, authentic standards, AromaOffice version 7.0 (Nishikawa Keisoku Co. Ltd., Tokyo, Japan), and AroChemBase version 7.0 (Alpha MOS, Toulouse, France) were used. The volatile compounds were identified based on three criteria: (1) by comparing the mass spectra of each compound with the NIST 8.0 mass spectra library, (2) by comparing the retention index with the literature data of AroChemBase, and (3) by comparing the mass spectra and retention time with an authentic standard. The AroChemBase module consists of a library of chemical compounds with name, formula, CAS number, molecular weight, Kovats retention index, sensory attributes, odor threshold, and related bibliography. For OA-LLE and SAFE, peak area ratios of the analyte to the internal standard (cyclohexanol) were used to calculate each concentration of volatiles in the extracts. In brief, $2 \mu \mathrm{L}$ of $0.5 \%(\mathrm{w} / \mathrm{v})$ cyclohexanol in dichloromethane was spiked into each of the extracts and authentic standard solutions $(200 \mu \mathrm{L})$. Some of the compounds were substituted with a compound that has a similar structure, and the amount was calculated as a semi-quantification.

\section{Enantioselective GC-MS for $\delta$-lactones}

The enantiomers of $\delta$-lactones in EVCO were analyzed using a 7890 A GC System gas chromatograph (Agilent Technologies, Inc.) coupled with a 5975 C inert XL MSD mass spectrometer detector (Agilent Technologies, Inc.) and a Supelco $\beta$-DEX 225 capillary column $(30 \mathrm{~m} \times 0.25 \mathrm{~mm}$, coated with a $0.25 \mu \mathrm{m}$ layer of liquid phase; Merck KGaA, Germany). The carrier gas was helium (99.995\%) and the flow rate was set at $1 \mathrm{~mL} \mathrm{~min}^{-1}$. A $1 \mu \mathrm{L}$ aliquot of the standard solution and the diluted extract obtained by OALLE (50-fold diluted extract for $\delta-C_{6}-C_{12}, 5$-fold diluted extract for $\delta-C_{14}$ and $\left.\delta-C_{16}\right)$ was injected. The oven temperature for $\delta-C_{6}-C_{12}$ was set at $90^{\circ} \mathrm{C}$ for $2 \mathrm{~min}$, increased to $160^{\circ} \mathrm{C}$ at $2^{\circ} \mathrm{C} \mathrm{min}{ }^{-1}$, maintained for $70 \mathrm{~min}$, increased to $230^{\circ} \mathrm{C}$ at $10^{\circ} \mathrm{C} \mathrm{min}-1$, and finally maintained at $230^{\circ} \mathrm{C}$ for $5 \mathrm{~min}$. The oven temperature for $\delta-C_{14}$ and $\delta-C_{16}$ was set at $90^{\circ} \mathrm{C}$ for $2 \mathrm{~min}$, increased to $160^{\circ} \mathrm{C}$ at $2^{\circ} \mathrm{C} \mathrm{min}{ }^{-1}$, maintained for $120 \mathrm{~min}$, increased to $230^{\circ} \mathrm{C}$ at $3{ }^{\circ} \mathrm{C}$ $\mathrm{min}^{-1}$, and finally maintained at $230^{\circ} \mathrm{C}$ for $5 \mathrm{~min}$. The splitless injection mode was at $230^{\circ} \mathrm{C}$. Mass spectra in the electron impact mode (EI) were generated at $70 \mathrm{eV}$ and the MSD transfer line temperature was set to $230^{\circ} \mathrm{C}$. The mass scan range was 30 to $400 \mathrm{~m} / \mathrm{z}$. Selected ion monitoring $(44,55,71$, and $99 \mathrm{~m} / \mathrm{z})$ was applied to describe the chromatograms and the enantiometric ratio of $\delta$-lactones. The peaks were identified by comparing the retention times and MS fragment pattern with those of pure racemic standards. The elution order of enantiomers was determined according to the literature ${ }^{41}$. The enantiomeric ratio of $\delta$-lactones contained in the extract from OA-LLE was also calculated from the peak areas obtained by an enantioselective GC-MS. The enantioselective GC-MS analysis was performed in triplicate.

\section{Log Pow value}

The log Pow value of volatile compounds was obtained from PubChem database (last access date: 27 May 2020).

\section{Odor activity value (OAV)}

The OAV of each volatile compound in the extract was calculated by dividing the concentration of a volatile compound by its threshold value in oil or water media. Threshold values were obtained from AroChemBase. Because the aroma extracts were obtained from $5 \mathrm{~g}$ of EVCO, the concentration of volatile compounds contained in the OA-LLE extract was converted to $\mathrm{mg} / \mathrm{kg}$ by multiplying by 200 .

\section{Sensory analysis}

The method was in accordance with Selli et al. with a minor modification ${ }^{42}$. In brief, the panelists were instructed to sniff and memorize the aroma of 
the reference sample, EVCO, and for the extract, to sniff the smelling strip odor and to determine the similarity and intensity of the odors. A smelling strip (Daimonji Paper Co., Ltd., Tokyo, Japan) was used for the representativeness test of the aromatic extract obtained by OA-LLE and SAFE. The extract $(15 \mu \mathrm{L})$ was applied to the extremity of the smelling strip. After $30 \mathrm{~s}$ (the time necessary for solvent evaporation), the smelling strip was given to a panelist. The panelists evaluated the quantity of the odor similarity and intensity of the aromatic extract by comparing with the odor of the reference. For the similarity test, a $100-\mathrm{mm}$ unstructured scale was used anchored with "far from the reference" on the left and "near to the reference" on the right. For the intensity test, a 100-mm unstructured scale was used anchored with "no odor" on the left and "very strong odor" on the right. The position of the sample on the unstructured scale was read as the distance in millimeters from the left anchor. Results were expressed as a mean score of the sensory perceptions of five panelists from our laboratory (one female and four males, 30 to 50 years old). The room temperature was set to $25^{\circ} \mathrm{C}$.

\section{Statistical analysis}

All statistical analyses were carried out using Microsoft Excel version 15.0.4963.1000 or JMP 14.3 provided by SAS Institute Inc. (Cary, NC, USA). Bartlett's test for homogeneity of variance was carried out to compare the variances of log Pow values. For sensory analysis, the mean scores were compared by the two-sided paired $t$-test, following a test for normality using the Shapiro-Wilk test. A significance level of each analysis was set to $p<0.05$.

\section{DATA AVAILABILITY}

The authors declare that all data supporting the findings of this study are available within the paper and its supplementary information.

Received: 11 June 2020; Accepted: 18 September 2020; Published online: 04 November 2020

\section{REFERENCES}

1. OECD/FAO, OECD-FAO Agricultural Outlook 2017-2026, OECD Publishing, Paris, https://doi.org/10.1787/agr_outlook-2017-en (2017).

2. Chandrashekar, J., Hoon, M. A., Ryba, N. J. P. \& Zuker, C. S. The receptors and cells for mammalian taste. Nature 444, 288-294 (2006).

3. Liu, D. L., Archer, N., Duesing, K., Hannan, G. \& Keast, R. Mechanism of fat taste perception: association with diet and obesity. Prog. Lipid Res. 63, 41-49 (2016).

4. Yin, W., Hewson, L., Linforth, R., Taylor, M. \& Fisk, I. D. Effects of aroma and taste, independently or in combination, on appetite sensation and subsequent food intake. Appetite 114, 265-274 (2017).

5. Arthur, C. L. \& Pawliszyn, J. Solid-phase microextraction with thermal desorption using fused-silica optical fibers. Anal. Chem. 62, 2145-2148 (1990).

6. Risticevic, S., Lord, H., Górecki, T., Arthur, C. L. \& Pawliszyn, J. Protocol for solidphase microextraction method development. Nat. Protoc. 5, 122-139 (2010).

7. Zhao, J. et al. Meta-analysis of genome-wide association studies provides insights into genetic control of tomato flavor. Nat. Commun. 10, 1534 (2019).

8. Lukić, I., Carlin, S., Horvat, I. \& Vrhovsek, U. Combined targeted and untargeted profiling of volatile aroma compounds with comprehensive two-dimensional gas chromatography for differentiation of virgin olive oils according to variety and geographical origin. Food Chem. 270, 403-414 (2019).

9. Giuffrè, A. M. et al. Volatile profiles of extra virgin olive oil, olive pomace oil, soybean oil and palm oil in different heating conditions. LWT Food Sci. Technol. 117, 108631 (2020).

10. Engel, W., Bahr, W. \& Schieberle, P. Solvent assisted flavour evaporation - a new and versatile technique for the careful and direct isolation of aroma compounds from complex food matrices. Eur. Food Res. Technol. 209, 237-241 (1999).

11. Peres, F. et al. Characterization of aroma compounds in Portuguese extra virgin olive oils from Galega Vulgar and Cobrançosa, cultivars using GC-O and GC × GCToFMS. Food Res. Int. 54, 1979-1986 (2013).

12. Sonmezdag, A. S., Kelebek, H. \& Selli, S. Pistachio oil (Pistacia vera L. Cv. Uzun): characterization of key odorants in a representative aromatic extract by GC-MSolfactometry and phenolic profile by LC-ESI-MS/MS. Food Chem. 240, 24-31 (2018).

13. Afoakwa, E. O., Paterson, A., Fowler, M. \& Ryan, A. Matrix effects on flavor volatiles release in dark chocolates varying in particle size distribution and fat content using GC-mass spectrometry and GC-olfactometry. Food Chem. 113, 208-215 (2009).
14. Jia, X. et al. Identification of key aroma-active compounds in sesame oil from microwaved seeds using E-nose and HS-SPME-GC $\times$ GC-TOF/MS. J. Food Biochem. 43, e12786 (2019).

15. Martins, N. et al. Revisiting $3 \mathrm{D}$ van Krevelen diagrams as a tool for the visualization of volatile profile of varietal olive oils from Alentejo region, Portugal. Talanta 207, 120276 (2020).

16. Suzuki, D. et al. A novel extraction method for aroma isolation from dark chocolate based on the oiling-out effect. Food Anal. Methods 12, 2857-2869 (2019).

17. Lin, F. M. \& Wilkens, W. F. Volatile flavor components of coconut meat. J. Food Sci. 35, 538-539 (1970).

18. Saittagaroon, S., Kawakishi, S. \& Namiki, M. Aroma constituents of roasted coconut. Agric. Biol. Chem. 48, 2301-2307 (1984).

19. Allen, R. R. Volatile flavor constituents in coconut oil. Chem. Ind. 36, 1560 (1965).

20. Pai, J. S., Lomanno, S. S. \& Nawar, W. W. Effect of heat treatments on the volatile composition of coconut oil. J. Am. Oil Chem. Soc. 56, 494-497 (1979).

21. Santos, J. E. R., Villarino, B. J., Zosa, A. R. \& Dayrit, F. M. Analysis of volatile organic compounds in virgin coconut oil and their sensory attributes. Philipp. J. Sci. 140, 161-171 (2011).

22. Mosandl, A. Enantioselective capillary gas chromatography and stable isotope ratio mass spectrometry in the authenticity control of flavors and essential oils. Food Rev. Int. 11, 597-664 (1995).

23. Obi, J. et al. Simple quantification of lactones in milk fat by solvent extraction using gas chromatography-mass spectrometry. J. Oleo Sci. 67, 941-948 (2018).

24. Schlutt, B., Moran, N., Schieberle, P. \& Hofmann, T. Sensory-directed identification of creaminess enhancing volatiles and semivolatiles in full fat cream. J. Agric. Food Chem. 55, 9634-9645 (2007).

25. Koppenhoefer, B. et al. Enantiomeric odor differences and gas chromatographic properties of flavors and fragrances. Perfum. Flavor. 19, 1-14 (1994).

26. Cagliero, C. et al. Fast headspace-enantioselective GC-mass spectrometricmultivariate statistical method for routine authentication of flavoured fruit food. Food Chem. 132, 1071-1079 (2012).

27. Dia, V. P., Garcia, V. V., Mabesa, R. C. \& Tecson-Mendoza, E. M. Comparative physicochemical characteristics of virgin coconut oil produced by different methods. Philipp. Agric. Sci. 88, 462-475 (2005).

28. Villarino, B. J., Dy, L. M. \& Lizada, C. C. Descriptive sensory evaluation of virgin coconut oil and refined, bleached and deodorized coconut oil. LWT Food Sci. Technol. 40, 193-199 (2007).

29. Varma, S. R. et al. In vitro anti-inflammatory and skin protective properties of Virgin coconut oil. J. Tradit. Complement. Med. 9, 5-14 (2019).

30. Rele, A. S. \& Mohile, R. B. Effect of mineral oil, sunflower oil, and coconut oil on prevention of hair damage. J. Cosmet. Sci. 54, 175-192 (2003).

31. Gasser, U. \& Grosch, W. Primary odorants of chicken broth. Z. Lebensm. Unters. Forsch. 190, 3-8 (1990).

32. Acree, T. H., Barnard, J. \& Cunningham, D. G. A procedure for the sensory analysis of gas chromatographic effluents. Food Chem. 14, 273-286 (1984).

33. Franklin, L. M. et al. Flavor and acceptance of roasted California almonds during accelerated storage. J. Agric. Food Chem. 66, 1222-1232 (2018).

34. Šuklje, K. et al. Unravelling wine volatile evolution during Shiraz grape ripening by untargeted HS-SPME-GC $\times$ GC-TOFMS. Food Chem. 277, 753-765 (2019).

35. Youssef, O. et al. Volatile compounds and compositional quality of virgin olive oil from Oueslati variety: Influence of geographical origin. Food Chem. 124, 1770-1776 (2011)

36. Cavalli, J. F., Fernandez, X., Lizzani-Cuvelier, L. \& Loiseau, A. M. Characterization of volatile compounds of French and Spanish virgin olive oils by HS-SPME: Identification of quality-freshness markers. Food Chem. 88, 151-157 (2004).

37. Kato, S. et al. Determination of triacylglycerol oxidation mechanisms in canola oil using liquid chromatography-tandem mass spectrometry. npj Sci. Food 2, 1 (2018).

38. Lee, S. et al. CD36 involvement in the olfactory perception of oleic aldehyde, an odour-active volatile compound, in mice. Biomed. Res. 38, 207-213 (2017).

39. Tsuzuki, S., Amitsuka, T., Okahashi, T., Kimoto, Y. \& Inoue, K. A search for CD36 ligands from flavor volatiles in foods with an aldehyde moiety: Identification of saturated aliphatic aldehydes with 9-16 carbon atoms as potential ligands of the receptor. J. Agric. Food Chem. 65, 6647-6655 (2017).

40. Tsuzuki, S. Higher straight-chain aliphatic aldehydes: Importance as odor-active volatiles in human foods and issues for future research. J. Agric. Food Chem. 67, 4720-4725 (2019).

41. Lehmann, D., Mass, B. \& Monsandl, A. Stereoisomeric flavour compounds LXIX: stereodifferentiation of $\delta(\gamma)$-lactones $C_{8}-C_{18}$ in dairy products, margarine and coconut. Z. Leb. Unters. Forsch. 201, 55-61 (1995).

42. Selli, S. et al. Characterization of the most odor-active volatiles of orange wine made from a Turkish cv. Kozan (Citrus sinensis L. Osbeck). J. Agric. Food Chem. 56, 227-234 (2008).

43. High, R., Bremer, P., Kebede, B. \& Eyres, G. T. Comparison of four extraction techniques for the evaluation of volatile compounds in spray-dried New Zealand sheep milk. Molecules 24, 1917 (2019). 


\section{ACKNOWLEDGEMENTS}

We would like to thank Professor Dennis Murphy of Ehime University for language editing.

\section{AUTHOR CONTRIBUTIONS}

D.S. and H.T. designed the study. D.S. wrote the initial draft of the manuscript. D.S. and Y.S. contributed to data collection. D.S. performed the statistical analysis. D.S., Y.S., H.K., T.K., and H.T. interpreted the results and proofread the manuscript. All authors read and approved the final paper.

\section{COMPETING INTERESTS}

The authors declare no competing interests.

\section{ADDITIONAL INFORMATION}

Supplementary information is available for this paper at https://doi.org/10.1038/ s41538-020-00079-8.

Correspondence and requests for materials should be addressed to D.S.
Reprints and permission information is available at http://www.nature.com/ reprints

Publisher's note Springer Nature remains neutral with regard to jurisdictional claims in published maps and institutional affiliations.

Open Access This article is licensed under a Creative Commons Attribution 4.0 International License, which permits use, sharing, adaptation, distribution and reproduction in any medium or format, as long as you give appropriate credit to the original author(s) and the source, provide a link to the Creative Commons license, and indicate if changes were made. The images or other third party material in this article are included in the article's Creative Commons license, unless indicated otherwise in a credit line to the material. If material is not included in the article's Creative Commons license and your intended use is not permitted by statutory regulation or exceeds the permitted use, you will need to obtain permission directly from the copyright holder. To view a copy of this license, visit http://creativecommons. org/licenses/by/4.0/.

(c) The Author(s) 2020 